\section{Hebelwirkung für die Umwelt}

\author{
Die ökologische Unternehmensbewertung hat sich als Informationsinstrument \\ für interessierte Investoren etabliert. Selbst Großanleger entdecken Umweltin- \\ formationen als Entscheidungskriferium. Bei der Beschaffung der Informationen \\ steht das Öko-Rating allerdings immer noch vor bürokratischen Hürden.
}

Von Dirk Reinbard

$\mathrm{G}$ egen Ende der sechziger Jahre entstand in den USA die moderne Bewegung des ethischen Investments. Beeinflußt durch den Vietnamkrieg, suchten Stiftungen und Universitäten nach moralisch akzeptablen Investitionsmöglichkeiten - dem sogenannten ,ethischen Investment". Es zeichnet sich dadurch aus, daß bei der Anlageentscheidung auch nicht-monetäre Aspekte wie zum Beispiel Aktivitäten in der Rüstungsproduktion berücksichtigt werden.

Im deutschsprachigen Raum bildete sich mit dem zunehmenden Umweltbewußtsein in den achtziger Jahren eine Form des ethischen Investments, die anders als in den USA einen Schwerpunkt auf ökologische Aspekte legte. Heute bieten mehrere Investmentfonds eine Kapitalanlage nach ökologischen Kriterien an. Dabei unterscheiden sich die ökologischen Anlagekriterien jedoch erheblich: Der „Hypo-Eco-Tech“ der Hypo-Bank zum Beispiel investiert nur in Unternehmen aus dem Umwelttechnologiesektor. Der „OekoSar“ der Schweizer Bank Sarasin hingegen wird nach einem Nachhaltigkeitsansatz gemanagt. Nur Unternehmen, die gleichzeitig den Umweltschutzgedanken fest in den Geschäftsbetrieb integriert haben und wirtschaftlich erfolgreich arbeiten, sind nach Auffassung der Fondsmanager in der Lage, nachhaltig $\mathrm{zu}$ wirtschaften und können in das Portfolio aufgenommen werden - egal aus welcher Branche.

\section{- Entstehung des Öko-Ratings}

Mit der zunehmenden ökologischen Ausrichtung von Anlageentscheidungen entstand der Bedarf nach der Erhebung, Auswertung, Interpretation und verständlichen Aufbereitung umweltbezogener Daten und Unternehmensinformationen durch eine unabhängige Institution - dem Öko-Rating.

Die ökom GmbH aus München entwickelte hierzu Ende 1993 einen Öko-Rating-Ansatz. Seither wurden nach dieser ständig überprüften und weiterentwickelten Methode mehr als 40 internationale börsennotierte Aktiengesellschaften bewertet. Die Ergebnisse der Ratings werden zum Teil in Börsenzeitschriften wie der Zeitschrift „Börse Online“ und dem Informationsdienst „öko-Invest“ veröffentlicht.

\section{Datenerhebung und -auswertung}

Nach einer Sichtung der Unterlagen des Unternehmens wie zum Beispiel Geschäftsberichten, Umweltberichten und Produktinformationen wird ein circa 30seitiger Fragebogen erstellt, der die wichtigsten umweltrelevanten Bereiche des Unternehmens abdeckt.

Die Angaben des Unternehmens werden anschließend durch Auswertung von Sekundärliteratur und externe Recherche überprüft und ergänzt. Hierbei kommen Einschätzungen und Fachwissen externer Fachleute aus Umwelt- und Wirtschaftsverbänden, Forschungsinstituten, Verbraucherinitiativen etc. zum Tragen.

Die einzelnen Untersuchungsbereiche (siehe Abbildung 1) werden untereinander gewichtet

\section{Abb. 1: Untersuchungskriterien}

Der Ratingansatz besteht aus drei Teilen, die mit jeweils unterschiedlicher Gewichtung zur Gesamtbewertung beitragen.

I. Umweltmanagement (Gewichtung $25 \%$ )

- Umweltziele, Umweltbeauftragte

- Umwelt-Audit, Öko-Bilanz, Öko-Controlling

- Umweltstandards im Ausland

- Kooperationen, Ausbildung, Büro- und Kantinenbereich

- Beschaffungsrichtlinien, Transport, Absatz

II. Produkt- und Dienstleisfungsentwicklung (Gewichtung 50\%) ten und Dienstleistungen

- Maßnahmen und Ziele der ökologisch orientierten

Dienstleistungsentwicklung
- Umweltverträglichkeitsprüfungen bei einzelnen Produk.

- Maßnahmen und Ziele der ökologisch orientierten Pro. und auf einer Skala von +5 bis -5 bewertet. Die Bewertung mit Null repräsentiert ein Unternehmen, welches über das gesetzlich geforderte Mindestmaß hinaus kein Umweltengagement zeigt. Negativ bewertete Unternehmen haben in der Vergangenheit gegen Gesetze verstoßen. Die Note +5 soll das aus ökologischer Sicht machbare Optimum darstellen.

\section{Probleme der ökologischen Unternehmensbewertung}

Die wichtigsten Problemfelder der ökologischen Unternehmensbewertung lassen sich wie folgt zusammenfassen:

- Feblende Kooperationsbereitschaft der zu bewertenden Unternehmen: Aufgrund der Tatsache, daß das Öko-Rating innerhalb des relativ kleinen Marktes des Öko-Investments Anwendung gefunden hat, ist der Bekanntheitsgrad gerade im Ausland sehr gering. Hinzu kommt die von Land zu Land unterschiedliche Sensibilität für die Umweltproblematik. Manche Unternehmen sehen deshalb nicht die Notwendigkeit eines Öko-Ratings und verweigern die Mitarbeit, so daß wichtige Informationen nicht erhoben werden können. Dies kann in der Regel nur durch Druck von seiten der Investoren verhindert werden.

- Unzureichende Datenbasis und Genauigkeit der Daten: Bislang stellen nur wenige Unternehmen der Öffentlichkeit Umweltdaten zur Verfügung. Selbst Daten, die nach gesetzlichen Vorschriften erhoben werden müssen, sind nur schwer zugänglich. Daran hat auch das

duktentwickung z. B.:

- Langlebigkeit

- Reparatur- und Recyclingfreundlichkeit

- Verwendung ökologischer Materialien

- Reduzierung des Verbrauchs von nicht erneuerbaren Ressourcen beim Gebrauch des Produktes

- Reduzierung schädlicher Emissionen beim Gebrouch des Produktes

III. Umweltkennzahlen (Gewichtung $\mathbf{2 5 \% \text { ) }}$ Trends und Vergleich mit den Vorjahreswerten der folgenden ouf Umsatz, Output oder Mitarbeiter bezogenen Umweltdaten:

- Energieverbrauch

- Wosserverbrauch

- Abwasser

- $\mathrm{NO} x / \mathrm{SO}_{x} / \mathrm{CO}_{2}$

- Abfallmenge und -zusammensetzung 
(c) 20I0 Authors; licensee IÖW and oekom verlag. This is an article distributed under the terms of the Creative Commons Attribution Non-Commercial No Derivates License (http://creativecommons.org/licenses/by-nc-nd/3.o/), which permits unrestricted use, distribution, and reproduction in any medium, provided the original work is properly cited. 